\title{
On-Axis Electrode Aberration Correctors for Scanning Electron/Ion Microscopes
}

\author{
A. Khursheed and W. K. Ang \\ Department of Electrical and Computer Engineering, National University of Singapore, Singapore.
}

This paper presents a method for correcting spherical and chromatic aberrations of scanning electron/ion microscopes through the use of on-axis electrode corrector units. Unlike previous annular systems, which either required insertion of a corrector unit into the objective lens gap [1], or involved the use of special off-axis lenses [2], the on-axis electrode corrector units proposed here do not require any change to the microscope column design. They, together with an annular aperture, replace a conventional scanning electron/ion microscope's final hole-aperture unit, as shown in Figure 1. Apart from this, no other modification to the microscope design is required.

Figure 2 shows electric and magnetic designs for a spherical aberration on-axis electrode corrector. The electrostatic corrector is similar to a coaxial cable layout with a zero volt on-axis wire electrode, where the electric field, neglecting the end fringe field effects, is inversely proportional to the radius. In the magnetic case, an on-axis current carrying wire generates a circular magnetic field (Ampere's Law) whose strength is also inversely proportional to the radius. In both cases, the strength of the corrector unit focusing lens action applied to the primary electron/ion beam decreases as a function of radius, opposite to the over-focusing action of the objective lens, whose strength increases with radius. In this way, the spherical aberration effect of an objective lens can be reduced. Obviously, these correctors are more effective with decreasing annular aperture width. Whether the corrector requires a divergent or convergent action on the primary beam depends on the incoming angle of the primary beam and the working distance, however, this is not the key to understanding the way the corrector compensates for the spherical aberration, compensation is achieved by its falling field strength as a function of radius.

Consider the simple electrostatic Einzel objective lens column shown in Figure 3. An annular aperture electrostatic corrector unit is located $5 \mathrm{~cm}$ from a $10 \mathrm{kV}$ field emission source, and is a distance of 20 $\mathrm{mm}$ from the first electrode of the objective lens. The middle electrode of the objective lens is set to 20 $\mathrm{kV}\left(V_{F}\right)$, which forms a focal point $6.53 \mathrm{~mm}$ beyond the last objective lens electrode (the working distance). The field emission gun tip is modelled to be $5 \mu \mathrm{m}$ high and has a radius of $50 \mathrm{~nm}$. Direct ray tracing simulations were carried out by the commercial software, Lorentz 2EM [3]. The program calculates fields having rotational symmetry (two dimensions), and neglects all three dimensional complications, such as end effects in the corrector unit. Any loss of beam current due to the radial supports at the corrector end plates are assumed to be small and neglected. Six rays at a single energy of $0.2 \mathrm{eV}$ for uniformly distributed emission angles from the cathode-tip were plot to go through the annular aperture, and the value of $V_{C}$ in the corrector unit was adjusted so that the RMS value of the final spot size was minimized. Figure 4 shows that the simulated optimized corrected spherical aberration radius as a function of final semi-angle for a 70-80 $\mu \mathrm{m}$ aperture, together with the uncorrected (conventional) spherical aberration for a hole aperture that gives the same current ( 0 to $38.73 \mu \mathrm{m}$ ). The RMS value of the corrected spherical aberration radius $(1.75 \mathrm{~nm})$ is typically around one order of magnitude lower than its conventional counterpart $(18.5 \mathrm{~nm})$ and is centered on a semi-angle of 9 mrad.

The correctors shown in Figure 2 have obvious applications in situations where spherical aberration is dominant (low diffraction and chromatic aberrations), such as in the Helium Ion Microscope [4]. 


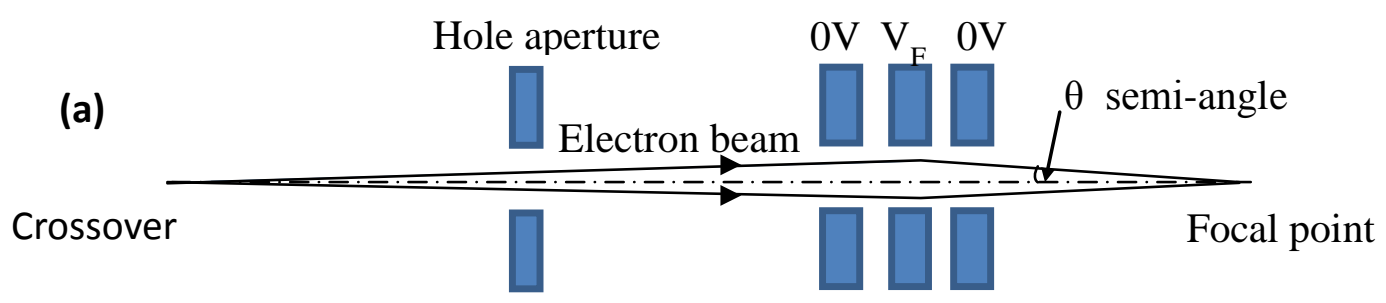

Objective lens

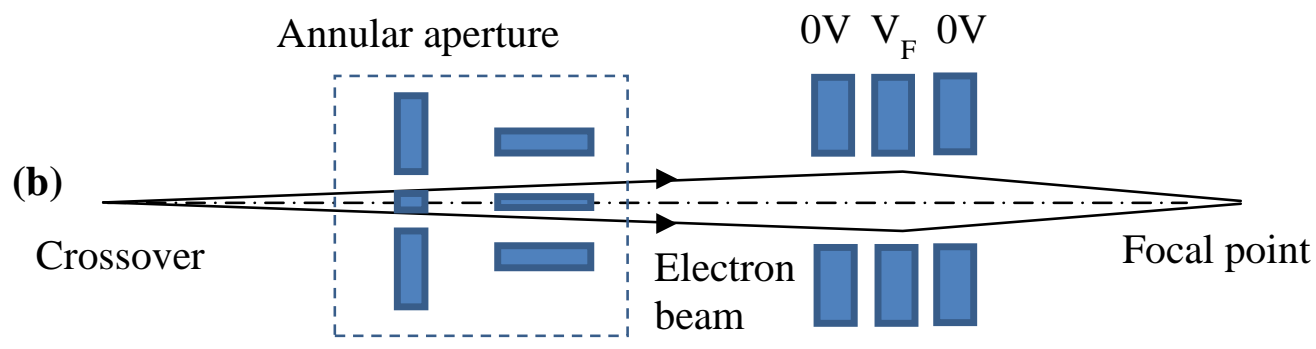

Corrector unit

Objective lens

Figure 1. Schematic diagram for the final stage of a probe forming electron/ion microscope (a) Conventional (b) With an on-axis aberration corrector
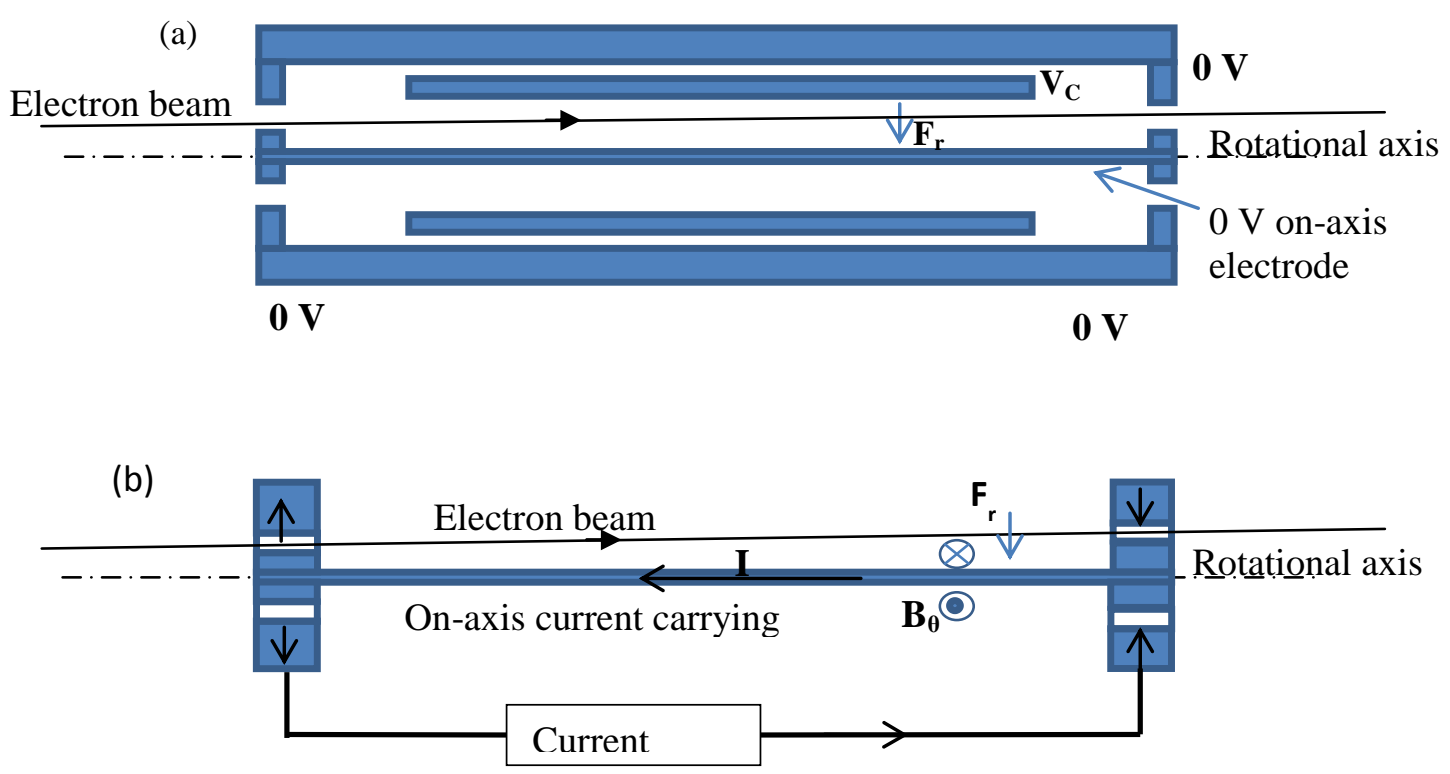

Figure 2. On-axis electrode spherical aberration correction unit. (a) Electrostatic (b) Magnetic 


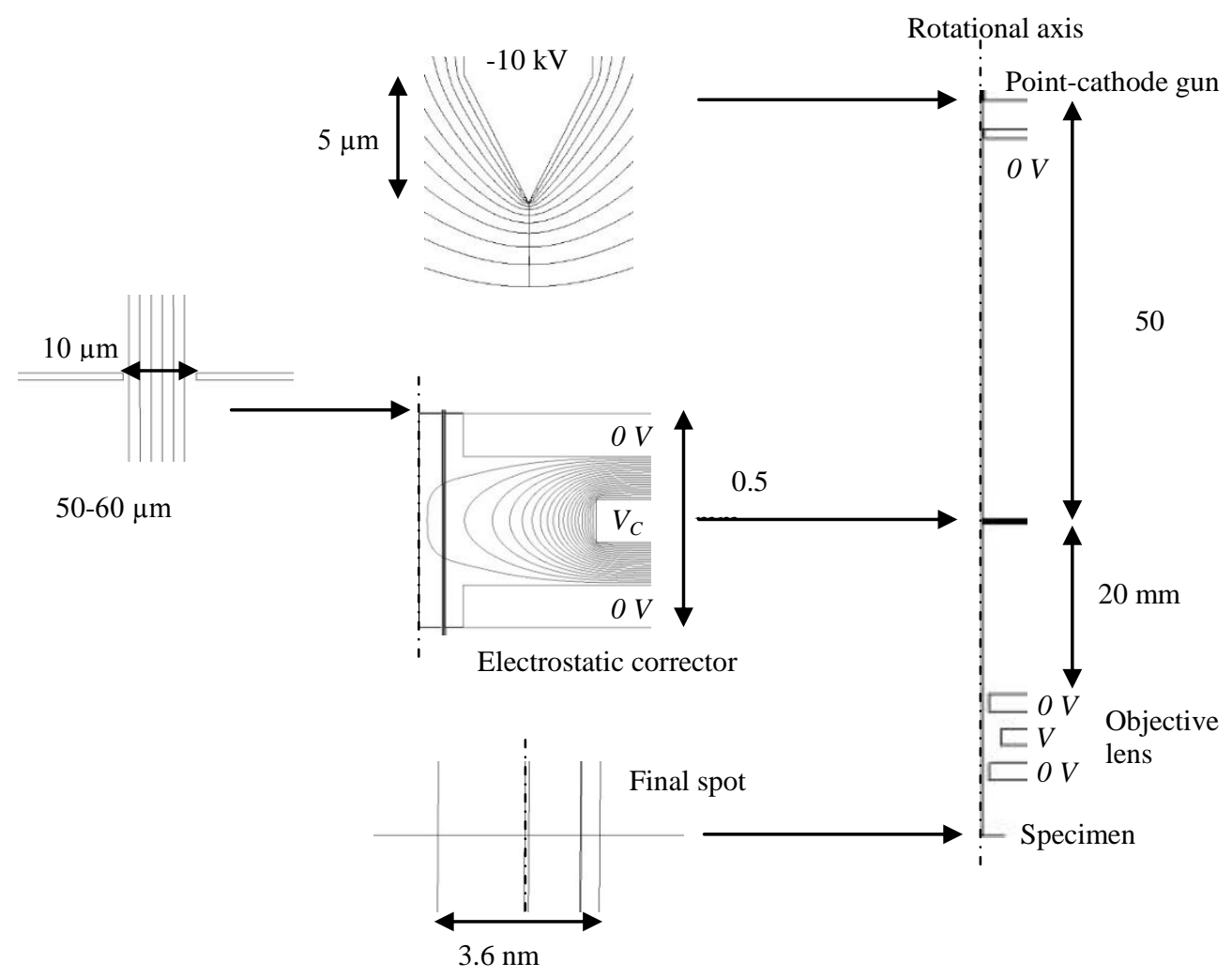

Figure 3. Direct ray tracing for an electric accelerating Einzel objective lens example. Six electrons pass through a 50 to $60 \mu \mathrm{m}$ annular aperture in uniform intervals and leave the cathode-tip of a field emitter. The cathode-tip radius is set at $50 \mathrm{~nm}$.

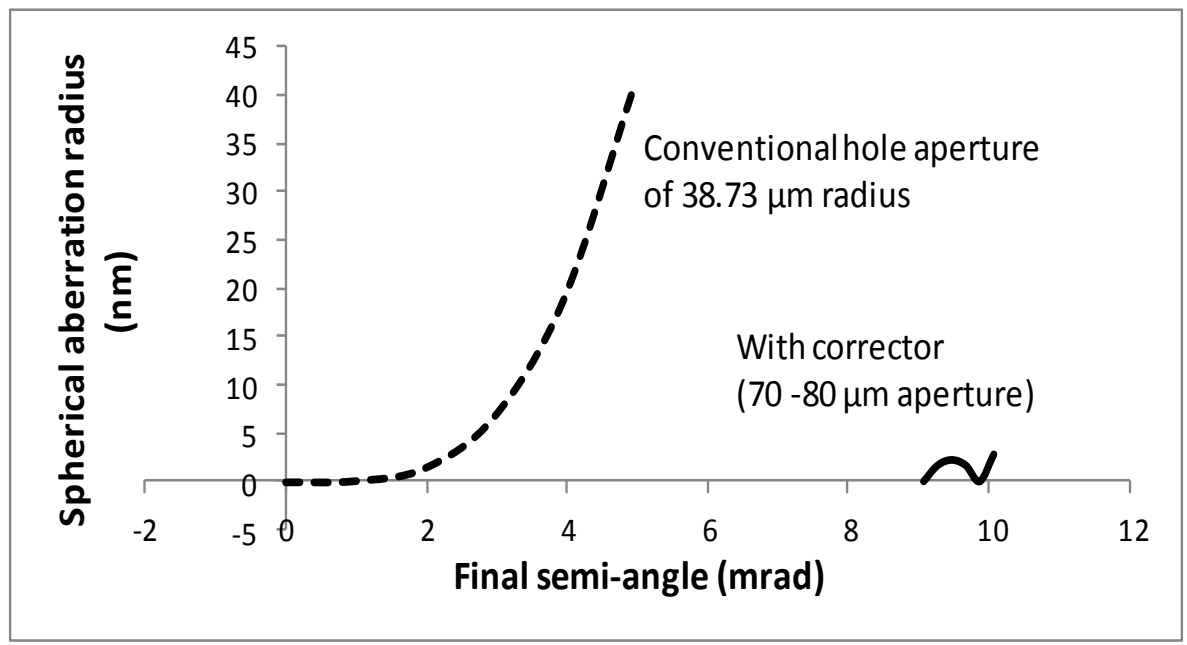

Figure 4. Simulated spherical aberration radius for a 70-80 $\mu \mathrm{m}$ aperture as a function of final semiangle in comparison to a conventional hole aperture for transmission of the same beam current. The corrector voltage was adjusted to minimise the RMS radius and found to be $V_{C}=-14.3$ volts.

The on-axis electrode concept also provides the possibility of correcting for the chromatic aberration of an objective lens. It can do this by superimposing electric and magnetic corrector fields, integrating them into one unit. In this case, the sign of the electric corrective force, $F_{e}$, points radially outwards, while the 
direction of the magnetic corrective force, $F_{m}$, points radially inwards, and they are adjusted so that the net force on the electrons or ions at a particular angle (such as those that pass through the minimum radius of the annular aperture) is zero, as indicated in Figure 5. Faster electrons will experience a greater inward force (since $F_{m}$ points towards the axis), while slower electrons will experience a radial force outwards. This is precisely opposite to the focusing action of the objective lens, where faster electrons experience a weaker focusing action than slower electrons. The mixed field on-axis electrode corrector should therefore be able to correct for the chromatic aberration of an objective lens.

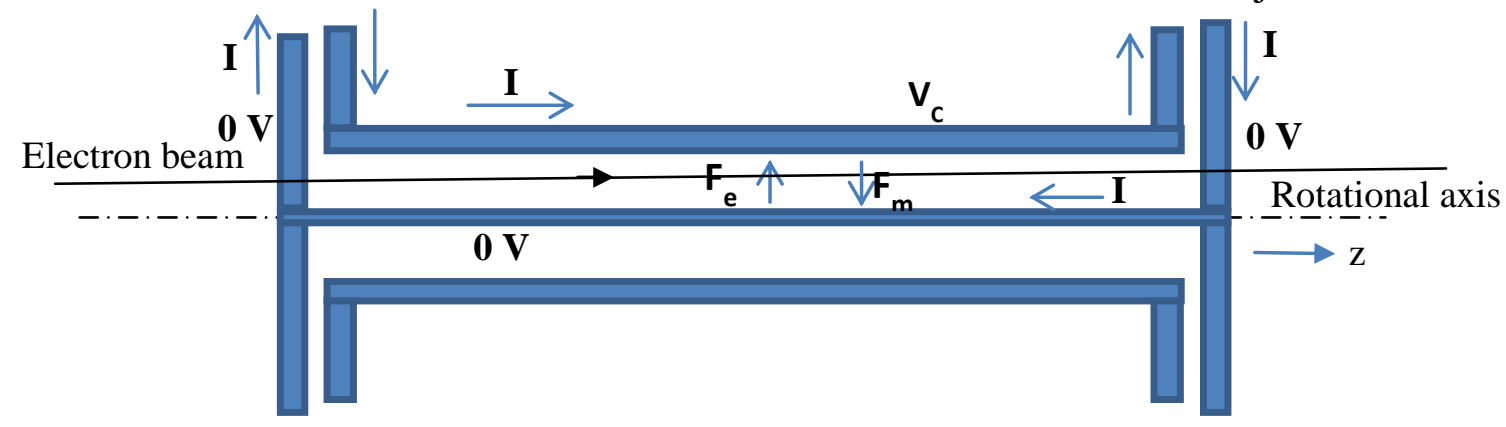

Figure 5. Chromatic aberration unit of superimposed magnetic and electrostatic fields

The effectiveness of chromatic aberration together with spherical aberration can be illustrated for the simple accelerating Einzel objective lens simulation example shown in Figure 6. In this case, a $1 \mathrm{keV}$ electron beam is produced by a simple point source located $100 \mathrm{~mm}$ from the spherical aberration correction unit, and the voltage in the Einzel lens is set to $2 \mathrm{kV}$, producing a final point of focus around $5.65 \mathrm{~mm}$ from the last lens electrode. A chromatic aberration corrector is placed directly after the spherical aberration corrector unit. The fields in both correctors are generated by simple analytical expressions. In the main body of each corrector an infinitely long coaxial solution is used for the electric case, and Ampere's Circuital Law around an infinitely long current carrying wire is used for the magnetic case. At the ends of each unit, a $\left[1+(d z / a)^{2}\right]^{-2}$ function fall-off is used, $d z$ is the distance from the end, and $a$ is a constant specifying how rapidly the functions falls. In this simple example, the diameters of the central wire and outer tube are $100 \mu \mathrm{m}$ and $200 \mu \mathrm{m}$ respectively, $a=50 \mu \mathrm{m}$ and an annular aperture whose pass radius varies from 70 to $80 \mu \mathrm{m}$ is specified. The simulation results of the probe size as a function of final semi-angle are shown in Figure 6 for the optimised potentials of $V_{C 1}=-$ $0.00217 \mathrm{~V}$ (in the spherical corrector), and $V_{C 2}=0.144 \mathrm{~V}$ (in the chromatic corrector). They predict that for FWHM energy spread of $0.5 \mathrm{eV}( \pm 0.25 \mathrm{eV})$, the average RMS radius of combined spherical and chromatic aberration correction is over a factor of 5 times smaller than for the uncorrected case (hole aperture giving the same probe current).

It should be noted that the excitation strength required in the chromatic aberration corrector is orders of magnitude higher than it is for the spherical aberration corrector. In practice, the length of the chromatic corrector will need to be made large enough in order to avoid heat density limits in the central current carrying wire. In this example, the $100 \mu \mathrm{m}$ diameter current carrying wire needs to conduct $55.4 \mathrm{~mA}$, which will produce a current density of $7.07 \mathrm{Amm}^{-2}$, exceeding the $4 \mathrm{Amm}^{-2}$ limit usually given for copper. However, the radial dimensions of the corrector can be scaled up. For a $140 \mu \mathrm{m}$ diameter central wire (and $280 \mu \mathrm{m}$ tube diameter), the current density falls to $3.59 \mathrm{Amm}^{-2}$. The maximum diameter of the central wire is obviously determined by the annular aperture inner diameter. For the chromatic aberration correction of ion beams, depending on the optics of the column, simply increasing the length 
of the chromatic corrector may not be possible, in which case, other means of obtaining the required current density may need to be investigated, such as the use of superconducting central wire electrodes.
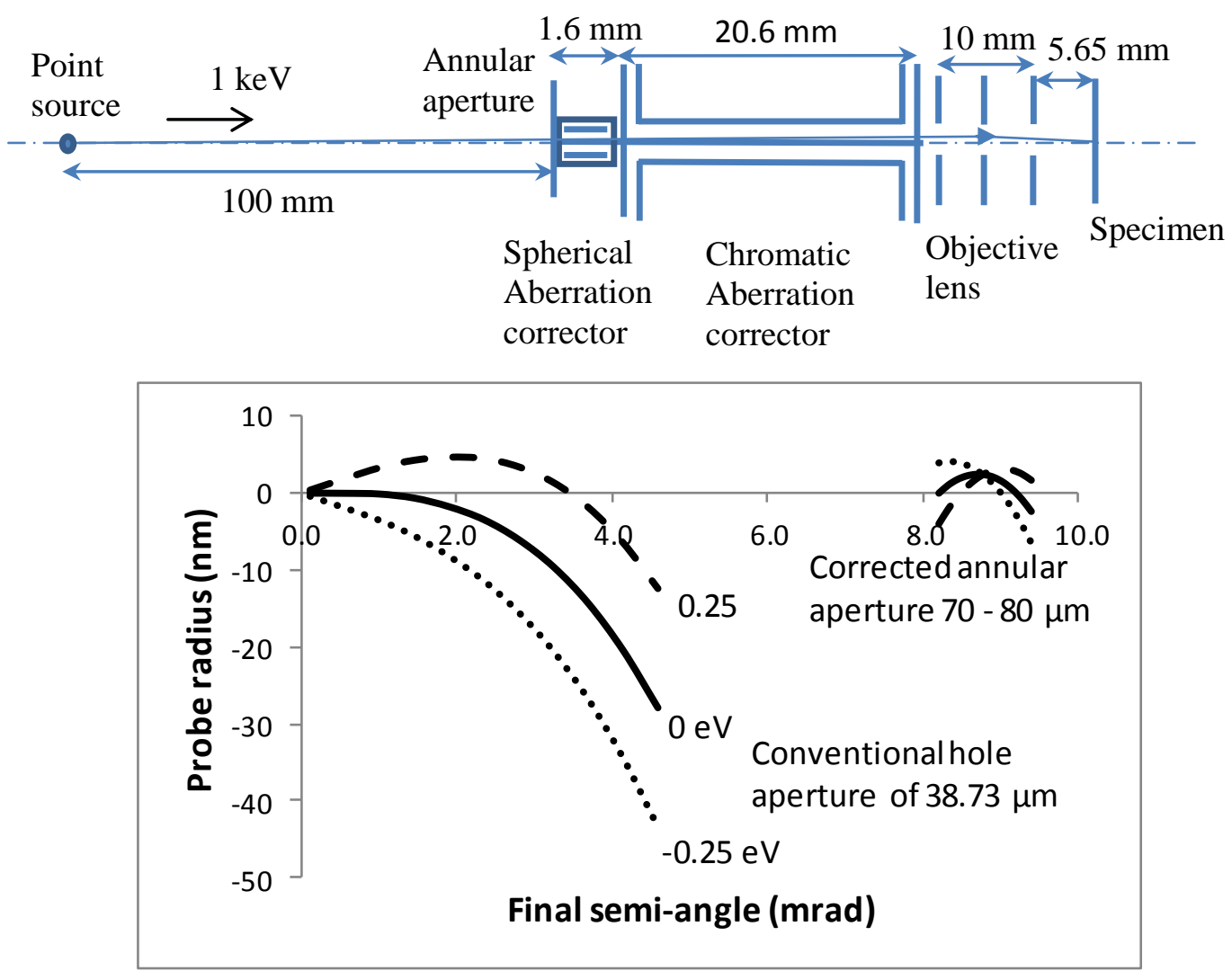

Figure 6. Simulation example of a combined chromatic and spherical aberration corrector for $1 \mathrm{keV}$ column test example with a FWHM energy spread of $0.5 \mathrm{eV}$

An approximate estimate of the diffraction aberration at radius $r$ at the focal plane, $\mathrm{M}(\mathrm{r})$, with wavenumber $k$ for a ring aperture having radii $R_{1}$ and $R_{2}$ projecting semi-angles $\theta_{1}$ and $\theta_{2}$ can be estimated by using the well-known Fraunhofer formula for circular shaped apertures,

$$
M(r)=\frac{M(0)}{\left(1-\varepsilon^{2}\right)}\left[\frac{2 J_{1}\left(X_{2}\right)}{X_{2}}-\frac{2 J_{1}\left(X_{1}\right)}{X_{1}} \varepsilon^{2}\right]
$$

where $\mathrm{M}(0)$ is the diffraction pattern value on the axis $(r=0), \mathrm{J}_{1}$ is the Bessel function of the first kind, order $1, \varepsilon=R_{1} / R_{2}, X_{2}=k r \sin \theta_{2}$, and $X_{1}=k r \sin \theta_{1}[5]$.

This function can be transformed into a normalised intensity pattern $[\mathrm{M}(\mathrm{r}) / \mathrm{M}(0)]^{2}$ and integrated as a function of radius to estimate the diffraction radius which contains $50 \%$ of the total current. This radius is plot in Figure 7 as a function of normalised ring width for different electron beam energies. As the ring width decreases, more of the current is transferred to the outer fringes, and the diffraction radius rises particularly sharply for the normalised width range between 0 and 0.1 . On the other hand, the diffraction radius decreases with average semi-angle, and in practice, a compromise needs to be found 
between deceasing the ring width to lower the chromatic and spherical aberrations, while at the same time enlarging it to prevent diffraction aberration from becoming too large. For the $10 \mathrm{keV}$ example column probe characteristics shown in Figure 4, (normalised ring width of 0.125 ), the 50\% current diffraction aberration radius is $2.85 \mathrm{~nm}$. For the $1 \mathrm{keV}$ example shown in Figure 6, the diffraction radius is $9.25 \mathrm{~nm}$ (70 to $80 \mu \mathrm{m}$ radius aperture), which drops to $5.26 \mathrm{~nm}$ for a 60 to $80 \mu \mathrm{m}$ radius annular aperture. Although the corrected RMS spherical aberration radius goes up to $6.33 \mathrm{~nm}$, it is still around a factor of 4 to 5 better than the probe radius predicted for the corresponding hole aperture at the same beam current.

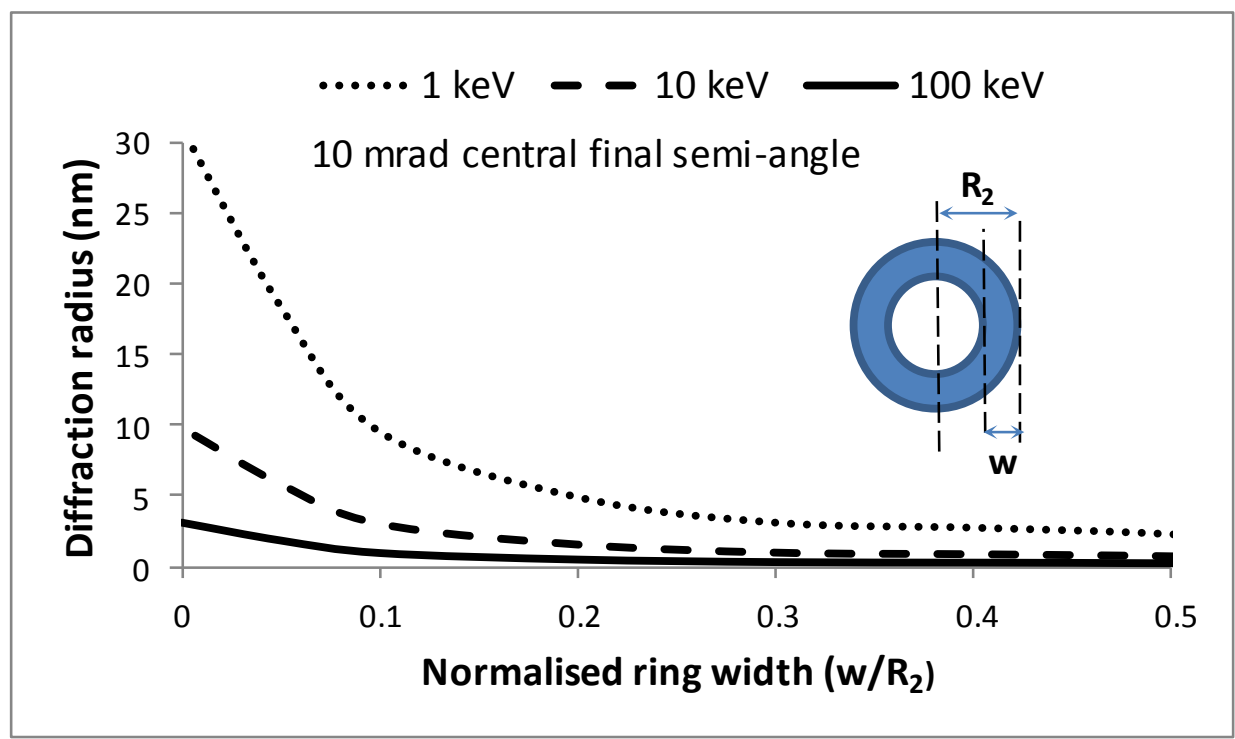

Figure 7. Calculated diffraction limited radius containing $50 \%$ of the probe current as a function of normalised ring width for different primary beam energies

Initial promising applications for the aberration correction method reported here include aberration correction for the Helium Ion Microscope, Focused Ion Beam columns, High Voltage Electron Beam Lithography instruments and Scanning Transmission Electron Microscopes. A US patent application of the aberration correction method described in this paper has been filed.

\section{References:}

[1] F Lenz and AP Wilska, Optik 24 (1966/67), p. 383.

[2] H Slätis and K Siegbahn, Ark. Fysik 1 (1949), p. 339.

[3] LORENTZ-2EM, Integrated Engineering Software Inc., Canada.

[4] BW Ward, JA Notte and NP Economou, J. Vac. Sci. Technol. B 24 (2006), p. 2871.

[5] JCH Spence in "High-Resolution Electron Microscopy", (Oxford University Press) p. 80.

[6] US patent application, U.S. Serial No. 62/041,208. 